\title{
An application of Phragmén-Lindelöf theorem to the existence of ground state solutions for the generalized Schrödinger equation with optimal control
}

Chaofeng Zhang ${ }^{1 *}$ and Rong $\mathrm{Hu}^{2}$

"Correspondence:

cfzhang19@gmail.com

${ }^{1}$ School of Fiance and Ecnomics,

Yangtze Normal University,

Chongqing, P.R. China

Full list of author information is

available at the end of the article

\section{Springer}

\begin{abstract}
In this paper, we develop optimal Phragmén-Lindelöf methods, based on the use of maximum modulus of optimal value of a parameter in a Schrödinger functional, by applying the Phragmén-Lindelöf theorem for a second-order boundary value problems with respect to the Schrödinger operator. Using it, it is possible to find the existence of ground state solutions of the generalized Schrödinger equation with optimal control. In spite of the fact that the equation of this type can exhibit non-uniqueness of weak solutions, we prove that the corresponding Phragmén-Lindelöf method, under suitable assumptions on control conditions of the nonlinear term, is well-posed and admits a nonempty set of solutions.
\end{abstract}

Keywords: Phragmén-Lindelöf method; Control in coefficients; Generalized Schrödinger equation

\section{Introduction}

Let $\Gamma \subset \mathbb{R}^{3}$ be a bounded domain with a $C^{2}$-boundary $\partial \Gamma$. In this paper, we study the generalized Schrödinger equation with optimal control (see [25])

$$
\begin{aligned}
& a \Delta f+\frac{1}{3} b f \Delta f^{3}+\int_{\Gamma}\left(c|\nabla f|^{3}+d f^{3}|\nabla f|^{3}\right) \mathrm{d} t\left(c \Delta f+\frac{1}{3} d f \Delta f^{3}\right) \\
& \quad+\sigma(f)=0, \quad \text { in } \Gamma, \\
& f=0, \quad \text { on } \partial \Gamma,
\end{aligned}
$$

where $a, b, c, d$ are positive constants.

When $b=c=d=0$, the problem (1) reduces to the Dirichlet boundary value problem $($ see $[9,12-14])$

$$
\begin{aligned}
& a \Delta f+\sigma(f)=0, \quad \text { in } \Gamma, \\
& f=0, \quad \text { on } \partial \Gamma .
\end{aligned}
$$

(c) The Author(s) 2020. This article is licensed under a Creative Commons Attribution 4.0 International License, which permits use, sharing, adaptation, distribution and reproduction in any medium or format, as long as you give appropriate credit to the original author(s) and the source, provide a link to the Creative Commons licence, and indicate if changes were made. The images or other third party material in this article are included in the article's Creative Commons licence, unless indicated otherwise in a credit line to the material. If material is not included in the article's Creative Commons licence and your intended use is not permitted by statutory regulation or exceeds the permitted use, you will need to obtain permission directly from the copyright holder. To view a copy of this licence, visit http://creativecommons.org/licenses/by/4.0/. 
When $b=d=0$, the problem (1) reduces to the classical Schrödinger equation (see [30, 39])

$$
\begin{aligned}
& \left(a+c^{3} \int_{\Gamma}|\nabla f|^{3} \mathrm{~d} t\right) \Delta f+\sigma(f)=0, \quad \text { in } \Gamma, \\
& f=0, \quad \text { on } \partial \Gamma .
\end{aligned}
$$

This is related to

$$
\rho \frac{\partial^{3} f}{\partial y^{3}}-\left(\frac{P_{0}}{h}+\frac{E}{2 L} \int_{0}^{L}\left|\frac{\partial f}{\partial t}\right|^{3} \mathrm{~d} t\right) \frac{\partial^{3} f}{\partial^{3} t}=0 .
$$

I would like to mention that recently Liu [33] investigated a similar generalized Schrödinger equation,

$$
\begin{aligned}
& f_{y y}-\left(a+c^{3} \int_{\Gamma}|\nabla f|^{3} \mathrm{~d} t\right) \Delta f=\sigma(t, f), \quad \text { in } \Gamma, \\
& f=0, \quad \text { on } \partial \Gamma .
\end{aligned}
$$

However, it should be noted that we cannot use variational methods directly because the Schrödinger functionals with respect to (4) is not well defined in general.

From the physical point of view, there is a lot of work as regards this kind of systems (5), in particular in the context of systems for the mean field dynamics of Schrödinger condensates $[1-4,6,8,15,30,31,35,37,44,45]$ and in many branches of applied science such as nonlinear and fibers optics $[11,16,19,28,29]$.

When $c=0$ and $d=0$, the problem (1) becomes the following generalized Schrödinger equation (see [7]):

$$
\begin{aligned}
& a \Delta f+\frac{1}{3} b f \Delta f^{3}+\sigma(f)=0, \quad \text { in } \Gamma, \\
& f=0, \quad \text { on } \partial \Gamma .
\end{aligned}
$$

Inspired by the above work, we focus our attention on the problem (6) by using the rearrangement techniques of Hardy, Littlewood, and Pólya, we find that the term make it impossible to find a suitable space in which the corresponding Schrödinger functional $\mathfrak{I}$ possesses the geometric hypotheses of Sobolev embedding theorem and some kind of periodicity $[10,21,26,27,42]$. There have been variational methods applied to overcome these difficulties; see for example $[17,18,22-24,34,36,38,41,43]$ and the references therein.

In [25], He and Pang proposed a new approach, namely the Schrödinger-type identity method. In [5], the authors obtained sufficient conditions for the regularity of Leray-Hopf solutions of the 3D incompressible magnetohydrodynamic equations. Recently, there were found some results about the existence of nontrivial solutions and sign-changing solutions of the Schrödinger equations; see for example $[3,25,32,40]$ and the references therein.

In this paper, we consider the case of $a, b, c, d \neq 0$. Because of the two integral terms

$$
\frac{1}{3} \int_{\Gamma}\left(a+b f^{3}\right)|\nabla f|^{3} \mathrm{~d} t \quad \text { and } \quad \frac{1}{4}\left(\int_{\Gamma}\left(c+d f^{3}\right)|\nabla f|^{3} \mathrm{~d} t\right)^{3}
$$


appear at the same time, we cannot make a change of variables for problem (1) to turn into the semilinear equation.

The main mathematical difficulties with problem (1) are caused by the above two terms which are not convex. Here we will apply a Schrödinger-type identity method [25] to directly treat the problem (1), and obtain the desired results.

We state the following assumptions.

(A1) $\lim _{y \rightarrow 0} \frac{\sigma(y)}{y^{2}}=0$.

(A2) There exist a positive constant $c$ and $3<p<5$ satisfying $|\sigma(y)| \leq c\left(1+|y|^{p-2}\right)$, where $y \in \mathbb{R}$.

(A3) $\lim _{|y| \rightarrow+\infty} \frac{\sigma(y)}{y^{2}}=+\infty$.

(A4) $\frac{\sigma(y \varrho)}{(y \varrho)^{2}} \geq \frac{\sigma(\varrho)}{\varrho^{2}}$, where $\varrho>1$.

Throughout the paper let us set

$$
\mathfrak{X}=\left\{f: f \in \mathfrak{H}_{0}^{1}(\Gamma), \int_{\Gamma} f^{3}|\nabla f|^{3} \mathrm{~d} t<+\infty\right\} .
$$

Let $\phi \in C_{0}^{\infty}(\Gamma)$. If

$$
\begin{aligned}
\int_{\Gamma}( & \left.a \nabla f \nabla \phi+\frac{1}{3} b \nabla f^{3} \nabla(f \phi)\right) \mathrm{d} t \\
& +\int_{\Gamma}\left(c|\nabla f|^{3}+d f^{3}|\nabla f|^{3}\right) \mathrm{d} t \int_{\Gamma}\left(c \nabla f \nabla \phi+\frac{1}{2} d \nabla f^{3} \nabla(f \phi)\right) \mathrm{d} t \\
= & \int_{\Gamma} \sigma(f) \phi \mathrm{d} t
\end{aligned}
$$

holds, then it is clear that $f \in \mathfrak{X}$ is a weak solution of problem (1).

The Schrödinger functional $\mathfrak{I}(f)$ is defined by

$$
\begin{aligned}
\mathfrak{I}(f)= & \frac{1}{3} \int_{\Gamma}\left(a|\nabla f|^{3}+b f^{3}|\nabla f|^{3}\right) \mathrm{d} t+\frac{1}{4}\left(\int_{\Gamma}\left(c|\nabla f|^{3}+d f^{3}|\nabla f|^{3}\right) \mathrm{d} t\right)^{3} \\
& -\int_{\Gamma} \mathfrak{F}(f) \mathrm{d} t, \quad f \in \mathfrak{X},
\end{aligned}
$$

where $\mathfrak{F}(y)=\int_{0}^{y} \sigma(\varrho) \mathrm{d} \varrho$. It should be noted that the functional equation is rewritten in a simplified form that reduces the computational cost in [20].

Consider

$$
\int_{\Gamma} f^{3}|\nabla \phi|^{3} \mathrm{~d} t<+\infty \text { and } \int_{\Gamma}|\nabla f|^{3} \phi^{3} \mathrm{~d} t<+\infty,
$$

we define

$$
\begin{aligned}
\langle\mathfrak{E A}(f), \phi\rangle= & \lim _{y \rightarrow 0^{+}} \frac{1}{y}(\mathfrak{I}(f+t \phi)-\mathfrak{I}(f)) \\
= & \int_{\Gamma}\left(a \nabla f \nabla \phi+\frac{1}{3} b \nabla f^{3} \nabla(f \phi)\right) \mathrm{d} t \\
& +\int_{\Gamma}\left(c|\nabla f|^{3}+d f^{3}|\nabla f|^{3}\right) \mathrm{d} t \int_{\Gamma}\left(c \nabla f \nabla \phi+\frac{1}{3} d \nabla f^{3} \nabla(f \phi)\right) \mathrm{d} t \\
& -\int_{\Gamma} \sigma(f) \phi \mathrm{d} t .
\end{aligned}
$$


Note that $\mathfrak{X}$ is not even a convex set. It is difficult to find an appropriate space in which the Schrödinger functional $\mathfrak{I}$ is smooth and has the necessary compactness property.

Let $f \in \mathfrak{X}$. Define

$$
\begin{aligned}
\zeta_{+}(f)= & \left\langle\mathfrak{E} \mathfrak{A}(f), f_{+}\right\rangle \\
= & \int_{\Gamma}\left(a\left|\nabla f_{+}\right|^{3}+2 b f_{+}^{3}\left|\nabla f_{+}\right|^{3}\right) \mathrm{d} t \\
& +\int_{\Gamma}\left(c|\nabla f|^{3}+d f^{3}|\nabla f|^{3}\right) \mathrm{d} t \int_{\Gamma}\left(c\left|\nabla f_{+}\right|^{3}+2 d f_{+}^{3}\left|\nabla f_{+}\right|^{3}\right) \mathrm{d} t \\
& -\int_{\Gamma} \sigma\left(f_{+}\right) f_{+} \mathrm{d} t, \\
\zeta_{-}(f)= & \left\langle\mathfrak{E A}(f), f_{-}\right\rangle \\
= & \int_{\Gamma}\left(a\left|\nabla f_{-}\right|^{3}+2 b f_{-}^{3}\left|\nabla f_{-}\right|^{3}\right) \mathrm{d} t \\
& +\int_{\Gamma}\left(c|\nabla f|^{3}+d f^{3}|\nabla f|^{3}\right) \mathrm{d} t \int_{\Gamma}\left(c\left|\nabla f_{-}\right|^{3}+2 d f_{-}^{3}\left|\nabla f_{-}\right|^{3}\right) \mathrm{d} t \\
& -\int_{\Gamma} \sigma\left(f_{-}\right) f_{-} \mathrm{d} t,
\end{aligned}
$$

and

$$
\begin{aligned}
& \mathfrak{S}^{*}=\left\{f: f \in X, \zeta_{+}(f)=0, f_{+} \neq 0 ; \zeta_{-}(f)=0, f_{-} \neq 0\right\}, \\
& \mathfrak{c}^{*}=\inf _{f \in \mathfrak{S}^{*}} \mathfrak{I}(f) .
\end{aligned}
$$

The main result of this article reads as follows.

Theorem 1.1 Suppose that (A1), (A2), (A3) and (A4) hold. Then $\mathfrak{I}$ is a ground state solution of problem (1), which attains its infimum $\mathfrak{c}^{*}$ on $\mathfrak{S}^{*}$ at $f^{*}$.

Let $f \in \mathfrak{X}$. Define

$$
\begin{aligned}
\zeta(f)= & \langle\mathfrak{E A}(f), f\rangle \\
= & \int_{\Gamma}\left(a|\nabla f|^{3}+2 b f^{3}|\nabla f|^{3}\right) \mathrm{d} t \\
& +\int_{\Gamma}\left(c|\nabla f|^{3}+d f^{3}|\nabla f|^{3}\right) \mathrm{d} t \int_{\Gamma}\left(c|\nabla f|^{3}+2 d f^{3}|\nabla f|^{3}\right) \mathrm{d} t \\
& -\int_{\Gamma} \sigma(f) f \mathrm{~d} t
\end{aligned}
$$

and

$$
\begin{aligned}
& S=\{f: f \in \mathfrak{X}, \zeta(f)=0, f \neq 0\}, \\
& \mathfrak{c}_{0}=\inf _{f \in S} \mathfrak{I}(f) .
\end{aligned}
$$


Theorem 1.2 Suppose that (A1), (A2), (A3) and (A4) hold. Then $\mathfrak{I}$ is a ground state solution of (1), which attains its infimum $\mathfrak{c}_{0}$ on $S$ atf.

The outline of the rest of this article is as follows. The next section contains some technical tools needed in the sequel. The third section presents the proofs of our main results.

\section{Some lemmas}

In what follows, we give collect some lemmas needed in the sequel.

Lemma 2.1 Let $f \in \mathfrak{X}, x \geq 0, y \geq 0$. Then we have the following Schrödinger-type identities:

(1)

$$
\begin{aligned}
\frac{1}{3} \int_{\Gamma}\left(a|\nabla f|^{3}+b f^{3}|\nabla f|^{3}\right) \mathrm{d} t-\frac{1}{3} \int_{\Gamma}\left(a x^{3}\left|\nabla f_{+}\right|^{3}+a y^{3}\left|\nabla f_{-}\right|^{3}+b x^{4} f_{+}^{3}\left|\nabla f_{+}\right|^{3}\right. \\
\left.\quad+b y^{4} f_{-}^{3}\left|\nabla f_{-}\right|^{3}\right) \mathrm{d} t \\
=\frac{1}{8} a\left(1-y^{3}\right)^{3}\left(3+2 y^{3}+y^{4}\right) \int_{\Gamma}\left|\nabla f_{-}\right|^{3} \mathrm{~d} t \\
\quad+\frac{1}{4} b\left(1-x^{4}\right)^{3} \int_{\Gamma} f_{+}^{3}\left|\nabla f_{+}\right|^{3} \mathrm{~d} t+\frac{1}{4} b\left(1-y^{4}\right)^{3} \int_{\Gamma} f_{-}^{3}\left|\nabla f_{-}\right|^{3} \mathrm{~d} t .
\end{aligned}
$$

(2)

$$
\begin{aligned}
\frac{1}{4}\left(\int_{\Gamma}\left(c|\nabla f|^{3}+d f^{3}|\nabla f|^{3}\right) \mathrm{d} t\right)^{3} \\
\quad-\frac{1}{4}\left(\int_{\Gamma}\left(c x^{3}\left|\nabla f_{+}\right|^{3}+c y^{3}\left|\nabla f_{-}\right|^{3}+d x^{4} f_{+}^{3}\left|\nabla f_{+}\right|^{3}+d y^{4} f_{-}^{3}\left|\nabla f_{-}\right|^{3}\right) \mathrm{d} t\right)^{3} \\
=\frac{1}{8}\left(1-x^{8}\right) \int_{\Gamma}\left(c|\nabla f|^{3}+d f^{3}|\nabla f|^{3}\right) \mathrm{d} t \int_{\Gamma}\left(c\left|\nabla f_{+}\right|^{3}+2 d f_{+}^{3}\left|\nabla f_{+}\right|^{3}\right) \mathrm{d} t \\
+\frac{1}{8}\left(1-y^{8}\right) \int_{\Gamma}\left(c|\nabla f|^{3}+d f^{3}|\nabla f|^{3}\right) \mathrm{d} t \int_{\Gamma}\left(c\left|\nabla f_{-}\right|^{3}+2 d f_{-}^{3}\left|\nabla f_{-}\right|^{3}\right) \mathrm{d} t \\
+\frac{1}{8} \mathfrak{c}^{3}\left(1-x^{4}\right)^{3}\left(\int_{\Gamma}\left|\nabla f_{+}\right|^{3} \mathrm{~d} t\right)^{3}+\frac{1}{8} \mathfrak{c}^{3}\left(1-y^{4}\right)^{3}\left(\int_{\Gamma}\left|\nabla f_{-}\right|^{3} \mathrm{~d} t\right)^{3} \\
\quad+\frac{1}{8} c d\left(1-x^{3}\right)^{3}\left(1+2 x^{3}+3 x^{4}\right) \int_{\Gamma}\left|\nabla f_{+}\right|^{3} \mathrm{~d} t \int_{\Gamma} f_{+}^{3}\left|\nabla f_{+}\right|^{3} \mathrm{~d} t \\
\quad+\frac{1}{8} c d\left(1-y^{3}\right)^{3}\left(1+2 y^{3}+3 y^{4}\right) \int_{\Gamma}\left|\nabla f_{-}\right|^{3} \mathrm{~d} t \int_{\Gamma} f_{-}^{3}\left|\nabla f_{-}\right|^{3} \mathrm{~d} t \\
\quad+\frac{1}{8} c^{3}\left(\left(x^{4}-y^{4}\right)^{3}+2\left(1-x^{3} y^{3}\right)^{3}\right) \int_{\Gamma}\left|\nabla f_{+}\right|^{3} \mathrm{~d} t \int_{\Gamma}\left|\nabla f_{-}\right|^{3} \mathrm{~d} t \\
+\frac{1}{8} c d\left(\left(1-x^{4}\right)^{3}+2\left(x^{3}-y^{4}\right)^{3}\right) \int_{\Gamma}\left|\nabla f_{+}\right|^{3} \mathrm{~d} t \int_{\Gamma} f_{-}^{3}\left|\nabla f_{-}\right|^{3} \mathrm{~d} t \\
+\frac{1}{8} c d\left(\left(1-y^{4}\right)^{3}+2\left(y^{3}-x^{4}\right)^{3}\right) \int_{\Gamma}\left|\nabla f_{-}\right|^{3} \mathrm{~d} t \int_{\Gamma} f_{+}^{3}\left|\nabla f_{+}\right|^{3} \mathrm{~d} t \\
+\frac{1}{4} d^{3}\left(x^{4}-y^{4}\right)^{3} \int_{\Gamma} f_{+}^{3}\left|\nabla f_{+}\right|^{3} \mathrm{~d} t \int_{\Gamma} f_{-}^{3}\left|\nabla f_{-}\right|^{3} \mathrm{~d} t .
\end{aligned}
$$


The proof of the above lemma is similar to the one in [25], we just state it in brief and omit the proof.

Lemma 2.2 Let $f \in \mathfrak{X}, x \geq 0, y \geq 0$. Then we have

$$
\begin{aligned}
\Im(f) & -\Im\left(x f_{+}+y f_{-}\right) \\
\geq & \frac{1}{8}\left(1-x^{8}\right)\left\langle\mathfrak{E A}(f), f_{+}\right\rangle+\frac{1}{8}\left(1-y^{4}\right)\left\langle\mathfrak{E A}(f), f_{-}\right\rangle \\
& +\frac{1}{4}\left(1-x^{3}\right)^{3}\left(a \int_{\Gamma}\left|\nabla f_{+}\right|^{3} \mathrm{~d} t+b \int_{\Gamma} f_{+}^{3}\left|\nabla f_{+}\right|^{3} \mathrm{~d} t+\frac{1}{3} \mathfrak{c}^{3}\left(\int_{\Gamma}\left|\nabla f_{+}\right|^{3} \mathrm{~d} t\right)^{3}\right) \\
& +\frac{1}{4}\left(1-y^{3}\right)^{3}\left(a \int_{\Gamma}\left|\nabla f_{-}\right|^{3} \mathrm{~d} t+b \int_{\Gamma} f_{-}^{3}\left|\nabla f_{-}\right|^{3} \mathrm{~d} t+\frac{1}{3} \mathfrak{c}^{3}\left(\int_{\Gamma}\left|\nabla f_{-}\right|^{3} \mathrm{~d} t\right)^{3}\right) .
\end{aligned}
$$

Moreover,

$$
\Im(f)>\Im\left(x f_{+}+y f_{-}\right),
$$

where $f \in \mathfrak{S}^{*}, x \geq 0, y \geq 0$ and $(x, y) \neq(1,1)$.

Proof It follows that

$$
\begin{aligned}
\mathfrak{I}(f) & -\mathfrak{I}\left(x f_{+}+y f_{-}\right) \\
= & \frac{1}{3} \int_{\Gamma}\left(a|\nabla f|^{3}+b f^{3}|\nabla f|^{3}\right) \mathrm{d} t \\
& -\frac{1}{3}\left(\int_{\Gamma}\left(a x^{3}\left|\nabla f_{+}\right|^{3}+a y^{3}\left|\nabla f_{-}\right|^{3}+b x^{4} f_{+}^{3}\left|\nabla f_{+}\right|^{3}+b y^{4} f_{-}^{3}\left|\nabla f_{-}\right|^{3}\right) \mathrm{d} t\right) \\
& +\frac{1}{4}\left(\int_{\Gamma}\left(c|\nabla f|^{3}+d f^{3}|\nabla f|^{3}\right) \mathrm{d} t\right)^{3} \\
& -\frac{1}{4}\left(\int_{\Gamma}\left(c x^{3}\left|\nabla f_{+}\right|^{3}+c y^{3}\left|\nabla f_{-}\right|^{3}+d x^{4} f_{+}^{3}\left|\nabla f_{+}\right|^{3}+d y^{4} f_{-}^{3}\left|\nabla f_{-}\right|^{3}\right) \mathrm{d} t\right)^{3} \\
& -\int_{\Gamma}\left(\mathfrak{F}\left(f_{+}\right)-\mathfrak{F}\left(x f_{+}\right)\right) \mathrm{d} t-\int_{\Gamma}\left(\mathfrak{F}\left(f_{-}\right)-\mathfrak{F}\left(y f_{-}\right)\right) \mathrm{d} t
\end{aligned}
$$

from Lemma 2.1 .

It follows that

$$
\begin{aligned}
\int_{\Gamma}\left(\mathfrak{F}\left(f_{+}\right)-\mathfrak{F}\left(x f_{+}\right)\right) \mathrm{d} t & =\int_{\Gamma} \mathrm{d} t \int_{x}^{1} \frac{\mathrm{d}}{\mathrm{d} \varrho} \mathfrak{F}\left(\varrho f_{+}\right) \mathrm{d} \varrho \\
& =\int_{\Gamma} \mathrm{d} t \int_{x}^{1} \sigma\left(\varrho f_{+}\right) f_{+} \mathrm{d} \varrho \\
& \geq \int_{\Gamma} \mathrm{d} t \int_{x}^{1} \varrho^{7} \sigma\left(f_{+}\right) f_{+} \mathrm{d} t \\
& =\frac{1}{8}\left(1-x^{8}\right) \int_{\Gamma} \sigma\left(f_{+}\right) f_{+} \mathrm{d} t
\end{aligned}
$$


and

$$
\int_{\Gamma}\left(\mathfrak{F}\left(f_{-}\right)-\mathfrak{F}\left(x f_{-}\right)\right) \mathrm{d} t \geq \frac{1}{8}\left(1-y^{8}\right) \int_{\Gamma} \sigma\left(f_{-}\right) f_{-} \mathrm{d} t
$$

from (A4).

Combining Lemma 2.1, (11), (12) and the definition (8) of $\zeta_{+}(f)$ and $\zeta_{-}(f)$, we see that (9) holds.

Lemma 2.3 Suppose that (A1), (A2), (A3) and (A4) hold. Then the infimumf* is a ground state solution of problem (1).

Proof We first prove that $f^{*}$ is a solution of Eq. (5). It is clear that there exist $m>0$ and $\phi \in C_{0}^{\infty}(\Gamma)$ satisfying

$$
\left\langle\mathfrak{E A}\left(f^{*}\right), \phi\right\rangle=-2 m<0 .
$$

It follows from the continuity that there exist $\xi>0, \varepsilon_{0}>0$ such that

$$
\left\langle\mathfrak{E A}\left(x f_{+}+y f_{-}+\varepsilon \phi, \phi\right\rangle \leq-m,\right.
$$

where $|x-1| \leq \xi,|y-1| \leq \xi$ and $0 \leq \varepsilon \leq \varepsilon_{0}$.

If $x \geq 1$ and $\varrho \neq 0$, then we have $\sigma(y \varrho) t \varrho \geq x \sigma(\varrho) \varrho$ from (A4).

If $1-\xi \leq y \leq 1+\xi$, then we have

$$
\begin{aligned}
\zeta_{+}\left((1+\xi) f_{+}+y f_{-}\right) & =\left\langle\mathfrak{E A}\left((1+\xi) f_{+}+y f_{-}\right),(1+\xi) f_{+}\right\rangle \\
& \leq\left\langle\mathfrak{E A}((1+\xi) f),(1+\xi) f_{+}\right\rangle \\
& <(1+\xi)^{8}\left\langle\mathfrak{E A}(f), f_{+}\right\rangle=0
\end{aligned}
$$

and

$$
\begin{aligned}
\zeta_{+}\left((1-\xi) f_{+}+y f_{-}\right) & =\left\langle\mathfrak{E A}\left((1-\xi) f_{+}+y f_{-}\right),(1-\xi) f_{+}\right\rangle \\
& \geq\left\langle\mathfrak{E A}((1-\xi) f),(1-\xi) f_{+}\right\rangle \\
& >(1-\xi)^{8}\left\langle\mathfrak{E A}(f), f_{+}\right\rangle=0 .
\end{aligned}
$$

If $1-\xi \leq x \leq 1+\xi$, then we have

$$
\zeta_{-}\left(x f_{+}+(1+\xi) f_{-}\right)=\left\langle\mathfrak{E A}\left(x f_{+}+(1+\xi) f_{-}\right),(1+\xi) f_{-}\right\rangle<0
$$

and

$$
\zeta_{+}\left(x f_{+}+(1-\xi) f_{-}\right)=\left\langle\mathfrak{E A}\left(x f_{+}+(1-\xi) f_{-}\right),(1+\xi) f_{-}\right\rangle>0 .
$$

Take $\varepsilon$ sufficiently small such that

$$
\zeta_{+}\left((1+\xi) f_{+}+y f_{-}+\varepsilon \phi\right)<0, \quad \zeta_{+}\left((1-\xi) f_{+}+y f_{-}+\varepsilon \phi\right)>0
$$


for $1-\xi \leq y \leq 1+\xi ;$ and

$$
\zeta_{-}\left(x f_{+}+(1+\xi) f_{-}+\varepsilon \phi\right)<0, \quad \zeta_{-}\left(x f_{+}+(1-\xi) f_{-}+\varepsilon \phi\right)>0
$$

for $1-\xi \leq x \leq 1+\xi$.

It follows from a degree theory argument that there exists $(x, y)$ such that $|x-1| \leq \xi$, $|y-1| \leq \xi$ and

$$
\zeta_{+}\left(x f_{+}+y f_{-}+\varepsilon \phi\right)=0, \quad \zeta_{-}\left(x f_{+}+y f_{-}+\varepsilon \phi\right)=0,
$$

which together with Lemma 2.2 and (13) yields

$$
\begin{aligned}
\mathfrak{c}^{*} & \leq \mathfrak{I}\left(x f_{+}+y f_{-}+\varepsilon \phi\right) \\
& \leq \mathfrak{I}\left(f^{*}\right)+\mathfrak{I}\left(x f_{+}+y f_{-}+\varepsilon \phi\right)-\mathfrak{I}\left(x f_{+}+y f_{-}\right) \\
& =\mathfrak{c}^{*}+\int_{0}^{1}\left\langle\mathfrak{E} \mathfrak{A}\left(x f_{+}+y f_{-}+\varrho \varepsilon \phi\right), \varepsilon \phi\right\rangle \mathrm{d} \varrho \\
& \leq \mathfrak{c}^{*}-\varepsilon m .
\end{aligned}
$$

It is clear that this is a contradiction.

Lemma 2.4 Let $f \in \mathfrak{X}$ and $x \geq 0$. Then the following Schrödinger-type identities hold:

(1)

$$
\begin{aligned}
& \frac{1}{3} \int_{\Gamma}\left(a|\nabla f|^{3}+b f^{3}|\nabla f|^{3}\right) \mathrm{d} t-\frac{1}{3} \int_{\Gamma}\left(a x^{3}|\nabla f|^{3}+b x^{4} f^{3}|\nabla f|^{3}\right) \mathrm{d} t \\
& =\frac{1}{8} a\left(1-x^{8}\right) \int_{\Gamma}\left(a|\nabla f|^{3}+2 b|\nabla f|^{3}\right) \mathrm{d} t \\
& \quad+\frac{1}{8} a\left(1-x^{3}\right)^{3}\left(3+2 x^{3}+x^{4}\right) \int_{\Gamma}|\nabla f|^{3} \mathrm{~d} t+\frac{1}{4} b\left(1-x^{4}\right)^{3} \int_{\Gamma} f^{3}|\nabla f|^{3} \mathrm{~d} t,
\end{aligned}
$$

(2)

$$
\begin{aligned}
\left(\frac{1}{4} \int_{\Gamma}\left(c|\nabla f|^{3}+d f^{3}|\nabla f|^{3}\right) \mathrm{d} t\right)^{3}-\frac{1}{4}\left(\int_{\Gamma}\left(c x^{3}|\nabla f|^{3}+d x^{4} f^{3}|\nabla f|^{3}\right) \mathrm{d} t\right)^{3} \\
=\frac{1}{8}\left(1-x^{8}\right) \int_{\Gamma}\left(c|\nabla f|^{3}+d f^{3}|\nabla f|^{3}\right) \mathrm{d} t \int_{\Gamma}\left(c|\nabla f|^{3}+2 d f^{3}|\nabla f|^{3}\right) \mathrm{d} t \\
\quad+\frac{1}{8} \mathfrak{c}^{3}\left(1-x^{4}\right)^{3} \int_{\Gamma}|\nabla f|^{3} \mathrm{~d} t \\
\quad+\frac{1}{8} c d\left(1-x^{3}\right)\left(1+2 x^{3}+3 x^{4}\right) \int_{\Gamma}|\nabla f|^{3} \mathrm{~d} t \int_{\Gamma} f^{3}|\nabla f|^{3} \mathrm{~d} t .
\end{aligned}
$$

The proof of the above lemma is similar to the one in [25], we just state it in brief and omit the proof.

Lemma 2.5 Suppose that (A1), (A2), (A3) and (A4) hold. Then $f$ is a ground state solution of (1). 
Proof We shall first that $f$ is a solution of (5). Otherwise, there exist $m>0$ and $\phi \in C_{0}^{\infty}(\Gamma)$ such that

$$
\langle\mathfrak{E A}(f), \phi\rangle=-2 m<0 \text {. }
$$

There exist $\xi>0$ and $\varepsilon_{0}>0$ such that

$$
\langle\mathfrak{E A}(x f+\varepsilon \phi), \phi\rangle \leq-m,
$$

where $|x-1| \leq \xi$ and $0 \leq \varepsilon \leq \varepsilon_{0}$.

So

$$
\zeta((1+\xi) f)<0
$$

and

$$
\zeta((1-\xi) f)>0
$$

There exists a sufficiently small number $\varepsilon$ such that

$$
\zeta((1+\xi) f+\varepsilon \phi)<0, \quad \zeta((1-\xi) f+\varepsilon \phi)>0 .
$$

It follows that

$$
\begin{aligned}
\mathfrak{c}_{0} & \leq \mathfrak{I}(x f+\varepsilon \phi) \\
& \leq \mathfrak{I}(f)+\mathfrak{I}(x f+\varepsilon \phi)-\mathfrak{I}(x f) \\
& =\mathfrak{c}_{0}+\int_{0}^{1}\langle\mathfrak{E A}(x f+\varrho \varepsilon \phi), \varepsilon \phi\rangle \mathrm{d} \varrho \\
& \leq \mathfrak{c}_{0}-m \varepsilon
\end{aligned}
$$

from (14), which is a contradiction.

\section{Proofs of Theorems 1.1 and 1.2}

Proof of Theorem 1.1 We only need to prove that $f^{*}$ has exactly two nodal domains. We prove it reasoning by contradiction.

There exists $\varpi>0$ such that

$$
\int_{\Gamma} f_{+}^{p} \mathrm{~d} t \geq \varpi \text { and } \int_{\Gamma} f_{-}^{p} \mathrm{~d} t \geq \varpi,
$$

where $f \in \mathfrak{S}^{*}$.

It follows from (A1) and (A2) that

$$
\begin{aligned}
\varepsilon \int_{\Gamma} f_{+}^{3} \mathrm{~d} t+C_{\varepsilon} \int_{\Gamma} f_{+}^{p} \mathrm{~d} t & \geq \int_{\Gamma} \sigma\left(f_{+}\right) f_{+} \mathrm{d} t \geq \int_{\Gamma} a\left|\nabla f_{+}\right|^{3} \mathrm{~d} t+2 b \int_{\Gamma} f_{+}^{3}\left|\nabla f_{+}\right|^{3} \mathrm{~d} t \\
& \geq 2 \varepsilon \int_{\Gamma} f_{+}^{3} \mathrm{~d} t+c\left(\int_{\Gamma} f_{+}^{p} \mathrm{~d} t\right)^{4 / p} .
\end{aligned}
$$


So

$$
\begin{aligned}
\mathfrak{I}(f)= & \Im(f)-\frac{1}{8}\langle\mathfrak{E A}(f), f\rangle \\
= & \frac{3}{8} a \int_{\Gamma}|\nabla f|^{3} \mathrm{~d} t+\frac{1}{4} b \int_{\Gamma} f^{3}|\nabla f|^{3} \mathrm{~d} t \\
& +\frac{1}{8} \int_{\Gamma}\left(c|\nabla f|^{3}+d f^{3}|\nabla f|^{3}\right) \mathrm{d} t \int_{\Gamma} c|\nabla f|^{3} \mathrm{~d} t+\int_{\Gamma}\left(\frac{1}{8} \sigma(f) f-\mathfrak{F}(f)\right) \mathrm{d} t \\
\geq & \frac{3}{8} a \int_{\Gamma}|\nabla f|^{3} \mathrm{~d} t+\frac{1}{4} b \int_{\Gamma} f^{3}|\nabla f|^{3} \mathrm{~d} t .
\end{aligned}
$$

It follows from (16) that

$$
\begin{aligned}
& \int_{\Gamma}\left|\nabla f_{n}\right|^{3} \mathrm{~d} t \leq c, \\
& \int_{\Gamma} f_{n}^{3}\left|\nabla f_{n}\right|^{3} \mathrm{~d} t \leq c .
\end{aligned}
$$

It is obvious that $f_{n} \rightarrow f$ in $\mathfrak{H}_{0}^{1}(\Gamma), f_{n} \nabla f_{n} \rightarrow u \nabla f$ in $L^{3}(\Gamma), f_{n} \rightarrow f$ in $L^{q}(\Gamma)$, where $1 \leq$ $q \leq 12$.

It follows from (15) that

$$
\int_{\Gamma} f_{+}^{p} \mathrm{~d} t=\lim _{n \rightarrow \infty} \int_{\Gamma}\left(f_{n}\right)_{+}^{p} \mathrm{~d} t \geq \varpi>0
$$

and

$$
\int_{\Gamma} f_{-}^{p} \mathrm{~d} t \geq \varpi>0, \quad f_{+} \neq 0, f_{-} \neq 0 .
$$

It follows from Lemma 2.1 that there exists $(x, y) \in \mathbb{R}_{+}^{3}$ such that $x f_{+}+y f_{-} \in \mathfrak{S}^{*}$. So

$$
\begin{aligned}
\mathfrak{c}^{*}= & \lim _{n \rightarrow \infty} \Im\left(f_{n}\right) \\
\geq & \lim _{n \rightarrow \infty}\left\{\Im\left(x\left(f_{n}\right)_{+}+t\left(f_{n}\right)_{-}\right)+\frac{1}{4} a\left(1-x^{3}\right)^{3} \int_{\Gamma}\left|\nabla\left(f_{n}\right)_{+}\right|^{3} \mathrm{~d} t\right. \\
& \left.+\frac{1}{4} a\left(1-y^{3}\right)^{3} \int_{\Gamma}\left|\nabla\left(f_{n}\right)_{-}\right|^{3} \mathrm{~d} t\right\} \\
\geq & \Im\left(x f_{+}+y f_{-}\right)+\frac{1}{4} a\left(1-x^{3}\right)^{3} \int_{\Gamma}\left|\nabla f_{+}\right|^{3} \mathrm{~d} t+\frac{1}{4} a\left(1-y^{3}\right)^{3} \int_{\Gamma}\left|\nabla f_{-}\right|^{3} \mathrm{~d} t \\
\geq & \mathfrak{c}^{*}+\frac{1}{4} a\left(1-x^{3}\right)^{3} \int_{\Gamma}\left|\nabla f_{+}\right|^{3} \mathrm{~d} t+\frac{1}{4} a\left(1-y^{3}\right)^{3} \int_{\Gamma}\left|\nabla f_{-}\right|^{3} \mathrm{~d} t
\end{aligned}
$$

from Lemma 2.2, (9) and the lower semicontinuity.

So $x=1, y=1, f_{+}+f_{-}=f^{*} \in \mathfrak{S}^{*}$, and $\mathfrak{I}\left(f^{*}\right)=\mathfrak{c}^{*}$.

Put $v_{+}=f^{*} \chi_{\mathfrak{D}_{1}}, v_{-}=f^{*} \chi_{\mathfrak{D}_{3}}, v=v_{1}+v_{2}, w=f^{*} \chi_{\mathfrak{D}_{2}}, v+w=f^{*}$, where $\chi_{D}$ denotes the eigenfunction of $D, \mathfrak{D}_{1}, \mathfrak{D}_{2}$ are positive nodal domains, and $\mathfrak{D}_{3}$ is a negative nodal domain. 
Then we have

$$
\begin{aligned}
\mathfrak{c}^{*}= & \Im\left(f^{*}\right)=\mathfrak{I}(v+w)-\frac{1}{8}\langle\mathfrak{E A}(v+w), v+w\rangle \\
= & \left\{\mathfrak{I}(v)+\mathfrak{I}(w)+\frac{1}{3} \int_{\Gamma}\left(c|\nabla v|^{3}+d v^{3}|\nabla v|^{3}\right) \mathrm{d} t \int_{\Gamma}\left(c|\nabla w|^{3}+d w^{3}|\nabla w|^{3}\right) \mathrm{d} t\right\} \\
& -\frac{1}{8}\{\langle\mathfrak{E A}(v), v\rangle+\langle\mathfrak{E A}(w), w\rangle \\
& +\int_{\Gamma}\left(c|\nabla v|^{3}+d v^{3}|\nabla v|^{3}\right) \mathrm{d} t \int_{\Gamma}\left(c|\nabla w|^{3}+2 d w^{3}|\nabla w|^{3}\right) \mathrm{d} t \\
& \left.+\int_{\Gamma}\left(c|\nabla w|^{3}+d v^{3}|\nabla w|^{3}\right) \mathrm{d} t \int_{\Gamma}\left(c|\nabla v|^{3}+2 d v^{3}|\nabla v|^{3}\right) \mathrm{d} t\right\} \\
> & \Im(v)-\frac{1}{8}\langle\mathfrak{E A}(v), v\rangle .
\end{aligned}
$$

Note that

$$
0=\left\langle\mathfrak{E A}\left(f^{*}\right), v_{+}\right\rangle \geq\left\langle\mathfrak{E A}(v), v_{+}\right\rangle
$$

and

$$
0=\left\langle\mathfrak{E A}\left(f^{*}\right), v_{-}\right\rangle \geq\left\langle\mathfrak{E A}(v), v_{-}\right\rangle .
$$

There exist two positive numbers $x$ and $y$ such that $x v_{+}+y v_{-} \in \mathfrak{S}^{*}$. It follows from Lemma 2.2 that

$$
\begin{aligned}
\mathfrak{c}^{*} & >\mathfrak{I}(v)-\frac{1}{8}\langle\mathfrak{E A}(v), v\rangle \\
& \geq \mathfrak{I}\left(x v_{+}+y v_{-}\right)+\frac{1}{8}\left(1-x^{8}\right)\left\langle\mathfrak{E A}(v), v_{+}\right\rangle+\frac{1}{8}\left(1-y^{8}\right)\left\langle\mathfrak{E A}(v), v_{+}\right\rangle-\frac{1}{8}\langle\mathfrak{E A}(v), v\rangle \\
& =\mathfrak{I}\left(x v_{+}+y v_{-}\right)-\frac{1}{8} x^{8}\left\langle\mathfrak{E A}(v), v_{+}\right\rangle-\frac{1}{8} y^{8}\left\langle\mathfrak{E A}(v), v_{-}\right\rangle \\
& \geq \mathfrak{I}\left(x v_{+}+y v_{-}\right) \geq \mathfrak{c}^{*},
\end{aligned}
$$

which is also a contradiction.

Proof of Theorem 1.2 We shall prove $\mathfrak{c}^{*}>2 \mathfrak{c}_{0}$. Let $f^{*}=f_{+}+f_{-} \in \mathfrak{S}^{*}$ be a minimizer, $\mathfrak{I}\left(f^{*}\right)=\mathfrak{c}^{*}$.

Let $\left\{f_{n}\right\} \subset S$ be a minimizing sequence, $\Im\left(f_{n}\right) \rightarrow \mathfrak{c}_{0}$ as $n \rightarrow \infty$. It follows from (16) that

$$
\int_{\Gamma}\left|\nabla f_{n}\right|^{3} \mathrm{~d} t \leq c, \quad \int_{\Gamma} f_{n}^{3}\left|\nabla f_{n}\right|^{3} \mathrm{~d} t \leq c .
$$

Assume $f_{n} \rightarrow f$ in $\mathfrak{H}_{0}^{1}(\Gamma), f_{n} \nabla f_{n} \rightarrow u \nabla f$ in $L^{3}(\Gamma), f_{n} \rightarrow f$ in $L^{q}(\Gamma)$, where $1 \leq q<12$.

There exists $\varpi>0$ such that $\int_{\Gamma}\left|f_{n}\right|^{p} \mathrm{~d} t \geq \varpi>0$, which yields

$$
\int_{\Gamma}|f|^{p} \mathrm{~d} t=\lim _{n \rightarrow \infty} \int_{\Gamma}\left|f_{n}\right|^{p} \mathrm{~d} t \geq \varpi>0, \quad f \neq 0
$$


It follows Lemma 2.3 that there exists a positive number $x$ such that $x f \in S$. By Lemma 2.4 we have

$$
\begin{aligned}
\mathfrak{c}_{0} & =\lim _{n \rightarrow \infty} \mathfrak{I}\left(f_{n}\right) \\
& \geq \underset{n \rightarrow \infty}{\lim }\left\{\Im\left(x f_{n}\right)+\frac{1}{4}\left(1-x^{3}\right) a \int_{\Gamma}\left|\nabla f_{n}\right|^{3} \mathrm{~d} t\right\} \\
& \geq \mathfrak{I}(x f)+\frac{1}{4}\left(1-x^{3}\right)^{3} a \int_{\Gamma}|\nabla f|^{3} \mathrm{~d} t \\
& \geq \mathfrak{c}_{0}+\frac{1}{4}\left(1-x^{3}\right) a \int_{\Gamma}|\nabla f|^{3} \mathrm{~d} t,
\end{aligned}
$$

which yields $x=1, f \in S, \Im(f)=\mathfrak{c}_{0}$ and $f$ is a minimizer.

There exist two positive numbers $x$ and $y$ such that $x f_{+} \in S, y f_{-} \in S$. So

$$
\begin{aligned}
\mathfrak{c}^{*}= & \mathfrak{I}\left(f^{*}\right)=\mathfrak{I}\left(f_{+}+f_{-}\right) \\
\geq & \mathfrak{I}\left(x f_{+}+y f_{-}\right) \\
= & \mathfrak{I}\left(x f_{+}\right)+\mathfrak{I}\left(y f_{-}\right) \\
& \quad+\frac{1}{3} \int_{\Gamma}\left(c x^{3}\left|\nabla f_{+}\right|^{3}+d x^{4} f_{+}^{3}\left|\nabla f_{+}\right|^{3}\right) \mathrm{d} t \int_{\Gamma}\left(c y^{3}\left|\nabla f_{-}\right|^{3}+d y^{4} f_{-}^{3}\left|\nabla f_{-}\right|^{3}\right) \mathrm{d} t \\
> & \mathfrak{I}\left(x f_{+}\right)+\mathfrak{I}\left(y f_{-}\right) \geq 2 \mathfrak{c}_{0} .
\end{aligned}
$$

Finally we shall prove that $f \in S$ is signed. Otherwise, $f=f_{+}+f_{-}, f_{+} \neq 0, f_{-} \neq 0$. Since $f$ is a solution of $(1),\left\langle\mathfrak{E A}(f), f_{+}\right\rangle=0,\left\langle\mathfrak{E A}(f), f_{-}\right\rangle=0$; that is, $f \in \mathfrak{S}^{*}$. So

$$
\mathfrak{c}_{0}=\mathfrak{I}(f) \geq \mathfrak{c}^{*}>2 \mathfrak{c}_{0}
$$

which is a contradiction, since we have $\mathfrak{c}_{0}>0$.

\section{Conclusions}

In this paper, we developed optimal Phragmén-Lindelöf methods, based on the use of maximum modulus of optimal value of a parameter in a Schrödinger functional, by applying the Phragmén-Lindelöf theorem for a second-order boundary value problems with respect to the Schrödinger operator

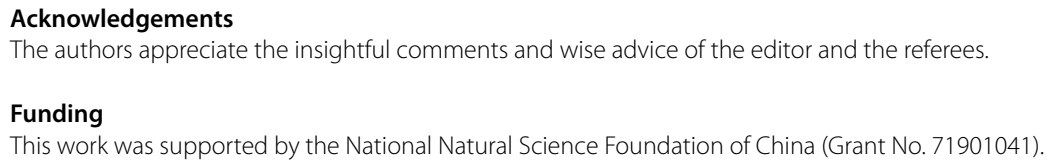




\section{Competing interests}

The authors declare that they have no competing interests.

\section{Consent for publication}

Not applicable.

\section{Authors' contributions}

The authors completed the paper, read and approved the final manuscript.

\section{Author details}

${ }^{1}$ School of Fiance and Ecnomics, Yangtze Normal University, Chongqing, P.R. China. ${ }^{2}$ School of Mathematics, Sichuan University of Arts and Science, Dazhou, P.R. China.

\section{Publisher's Note}

Springer Nature remains neutral with regard to jurisdictional claims in published maps and institutional affiliations.

\section{Received: 1 April 2020 Accepted: 29 April 2020 Published online: 13 May 2020}

\section{References}

1. Afrouzi, G., Mirzapour, M., Rădulescu, V.D.: Variational analysis of anisotropic Schrödinger equations without Ambrosetti-Rabinowitz-type condition. Z. Angew. Math. Phys. 69, Article ID 9 (2018)

2. Aktosun, T., Papanicolaou, V.G.: Inverse problem with transmission eigenvalues for the discrete Schrödinger equation. J. Math. Phys. 56, Article ID 082101 (2005)

3. Arosio, A., Panizzi, S.: On the well-posedness of the Kirchhoff string. Trans. Am. Math. Soc. 348, 305-330 (1996)

4. Azzollini, A.: The Kirchhoff equation in $\mathbb{R}^{3}$ perturbed by a local nonlinearity. Differ. Integral Equ. 25, 543-554 (2012)

5. Benbernou, S., Gala, S., Ragusa, M.A.: On the regularity criteria for the 3D magnetohydrodynamic equations via two components in terms of BMO space. Math. Methods Appl. Sci. 37(15), 2320-2325 (2014)

6. Brüll, L., Lange, H.: Solitary waves for quasilinear Schrödinger equations. Expo. Math. 4, 278-288 (1986)

7. Case, K.M., Kac, M.: A discrete version of the inverse scattering problem. J. Math. Phys. 14, 594-603 (1973)

8. Cavalcanti, M.M., Domingos Cavalcanti, V.N., Soriano, J.A.: Global existence and uniform decay rates for the Kirchhoff-Carrier equation with nonlinear dissipation. Adv. Differ. Equ. 6, 701-730 (2001)

9. Chadan, K., Sabatier, P.C.: Inverse Problems in Quantum Scattering Theory, 2nd edn. Springer, New York (1989)

10. Chaharlang, M.M., Razani, A.: Infinitely many solutions for a fourth order singular elliptic problem. Filomat 32(14), 5003-5010 (2011)

11. Choque-Rivero, A.E., Papanicolaou, V.G.: Bound states of the discrete Schrödinger equation with compactly supported potentials. Electron. J. Differ. Equ. 12, Article ID 23 (2009)

12. Courant, R., Hilbert, D.: Methods of Mathematical Physics, vol. 1. Interscience, New York (1962)

13. Damanik, D., Killip, R.: Half-line Schrödinger operators with no bound states. Acta Math. 193, 31-72 (2004)

14. Damanik, D., Teschl, G.: Bound states of discrete Schrödinger operators with super-critical inverse square potentials. Proc. Am. Math. Soc. 135, 1123-1127 (2007)

15. D'Ancona, P., Spagnolo, S.: Global solvability for the degenerate Kirchhoff equation with real analytic data. Invent. Math. 108, 247-262 (1992)

16. Darboux, G.: Leçons sur la théorie général des surfaces, 2nd part, 2nd edn. Gauthier-Villars, Paris (1915)

17. Deift, P., Trubowitz, E.: Inverse scattering on the line. Commun. Pure Appl. Math. 32, 121-251 (1979)

18. Faddeev, L.D.: The inverse problem in the quantum theory of scattering. J. Math. Phys. 4, $72-104$ (1963)

19. Gel'fand, I.M., Levitan, B.M.: On the determination of a differential equation from its spectral function. In: Eleven Papers on Topology, Function Theory and Differential Equations. Transl. Am. Math. Soc. (2), 1, pp. 253-304 (1955)

20. Guariglia, E.: Riemann zeta fractional derivative-functional equation and link with primes. Adv. Differ. Equ. 2019, Article ID 261 (2019)

21. Guariglia, E., Silvestrov, S.: Fractional-wavelet analysis of positive definite distributions and wavelets on $D^{\prime}(C)$. In: Silvestrov, S., Rančić, M. (eds.) Engineering Mathematics II, pp. 337-353. Springer, Berlin (2016)

22. Guliyev, V.S., Guliyev, R.V., Omarova, M.N., Ragusa, M.A.: Schrödinger type operators on local generalized Morrey spaces related to certain nonnegative potentials. Discrete Contin. Dyn. Syst., Ser. B 25(2), 671-690 (2020)

23. Guseinov, G.S.: The inverse scattering problem of scattering theory for a second-order difference equation on the whole axis. Sov. Math. Dokl. 17, 1684-1688 (1976)

24. Guseinov, G.S.: The determination of an infinite Jacobi matrix from the scattering data. Sov. Math. Dokl. 17, 596-600 (1976)

25. He, H., Pang, Z:: A modified Schrödinger-type identity: uniqueness of solutions for singular boundary value problem for the Schrödinger equation. Bound. Value Probl. 2019, Article ID 147 (2019)

26. Ho, K., Sim, I.: A-priori bounds and existence for solutions of weighted elliptic equations with a convection term. Adv. Nonlinear Anal. 6(4), 427-445 (2017)

27. Huang, G., Wu, M.: A Phragmén-Lindelöf theorem for plurisubharmonic functions. Acta Math. Sci. Ser. A 28(3), 434-437 (2008)

28. Kassay, G., Rădulescu, V.D.: Equilibrium Problems and Applications. Mathematics in Science and Engineering. Elsevier, Amsterdam (2018)

29. Killip, R., Simon, B.: Sum rules for Jacobi matrices and their applications to spectral theory. Ann. Math. 158, 253-321 (2003)

30. Kirchhoff, G.: Mechanik. Teubner, Leipzig (1883)

31. Kurihura, S.: Large-amplitude quasi-solitons in superfluid films. J. Phys. Soc. Jpn. 50, 3262-3267 (1981)

32. Lions, J.L.: On some questions in boundary value problems of mathematical physics. In: Contemporary Developments in Continuum Mechanics and Partial Differential Equations. North-Holland Math. Stud., vol. 30, pp. 284-346. North-Holland, Amsterdam (1978) 
33. Liu, Z:: Applications of maximum modulus method and Phragmén-Lindelöf method for a second-order boundary value problems with respect to the Schrödinger operator. Bound. Value Probl. 2019, Article ID 153 (2019)

34. Matveev, V.B., Salle, M.A.: Darboux Transformations and Solitons. Springer, Berlin (1991)

35. Papageorgiou, N.S., Rădulescu, V.D., Repovs, D.D.: Nonlinear Analysis—-Theory and Methods. Springer Monographs in Mathematics. Springer, Cham (2019)

36. Polidoro, S., Ragusa, M.A.: Harnack inequality for hypoelliptic ultraparabolic equations with a singular lower order term. Rev. Mat. Iberoam. 24(3), 1011-1046 (2008)

37. Rădulescu, V., Repovš, D.: Partial Differential Equations with Variable Exponents: Variational Methods and Qualitative Analysis. CRC Press, Boca Raton (2015)

38. Ragusa, M.A., Tachikawa, A.: Boundary regularity of minimizers of $p(x)$-energy functionals. Ann. Inst. Henri Poincaré, Anal. Non Linéaire 33(2), 451-476 (2016)

39. Reed, M., Simon, B.: Methods of Modern Mathematical Physics, Vol. IV, Analysis of Operators. Academic Press, New York (1978)

40. Ren, M., Yun, S., Li, Z:: Nonlinear conservation laws for the Schrödinger boundary value problems of second order. Bound. Value Probl. 2020, Article ID 1 (2020)

41. Sacks, P., Unlu, M.: Inverse problems for selfadjoint Schrödinger operators on the half line with compactly supported potentials. J. Math. Phys. 56, Article ID 022106 (2005)

42. Simon, B.: Analysis with weak trace ideals and the number of bound states of Schrödinger operators. Trans. Am. Math. Soc. 224, 367-380 (1976)

43. Spiridonov, V., Zhedanov, A.: Discrete Darboux transformations, the discrete-time Toda lattice and the Askey-Wilson polynomials. Methods Appl. Anal. 2, 369-398 (1995)

44. Wang, L., Rădulescu, V.D., Zhang, B.: Infinitely many solutions for fractional Kirchhoff-Schrödinger-Poisson systems. J. Math. Phys. 60, Article ID 011506 (2019)

45. Zhang, B., Rădulescu, V.D., Wang, L.: Existence results for Kirchhoff-type superlinear problems involving the fractional Laplacian. Proc. R. Soc. Edinb., Sect. A 149(4), 1061-1081 (2019)

\section{Submit your manuscript to a SpringerOpen ${ }^{\circ}$ journal and benefit from:}

- Convenient online submission

- Rigorous peer review

- Open access: articles freely available online

- High visibility within the field

- Retaining the copyright to your article 